\title{
Thermodynamic Fingerprints of Disorder in Flux Line Lattices and other Glassy Mesoscopic Systems
}

\author{
Thorsten Emig and Mehran Kardar \\ Physics Department, Massachusetts Institute of Technology, Cambridge, MA 02139, USA
}

(November 21, 2018)

\begin{abstract}
We examine probability distributions for thermodynamic quantities in finite-sized random systems close to criticality. Guided by available exact results, a general ansatz is proposed for replicated free energies, which leads to scaling forms for cumulants of various macroscopic observables. For the specific example of a planar flux line lattice in a two dimensional superconducting film near $H_{\mathrm{c} 1}$, we provide detailed results for the statistics of the magnetic flux density, susceptibility, heat capacity, and their cross-correlations.
\end{abstract}

PACS numbers: 74.60.Ge, 75.10.Nr, 73.23.-b

Impurities in a sample are expected to modify various measurements, making it desirable to characterize the probability distribution functions (PDFs) for the outcomes. These PDFs may provide important insight about the underlying physics; as in the case of universal conductance fluctuations in mesoscopic circuits, a subject of much recent investigation [1]. Here we consider the signature of impurities in thermodynamic systems at equilibrium. At one extreme, microscopic quantities such as two-point correlation functions are quite sensitive to disorder; in some cases their PDFs exhibiting complicated multiscaling behavior [2]. On the other hand, the free energy, and other macroscopic properties, are expected to be self-averaging, converging to fixed values in an infinite system. If all correlation lengths in the system are finite, the PDFs for a mesoscopic (finite-sized) sample should be governed by the central limit theorem. We thus focus on systems with long-range correlations, such as close to a critical point, or in a flux line (FL) lattice.

In the most interesting cases, disorder is relevant, leading to novel correlations distinct from the pure case. However, due the difficulty of characterizing collective behavior in such glassy disordered systems [3], little is known about the corresponding PDFs. Aharony and Harris [ 1 recently studied the PDFs of thermodynamic observables, near critical points with relevant randomness, in finite-sized random systems. They find a lack of self-averaging, and universal, non-Gaussian PDFs. There are also some results for elastic manifolds pinned by impurities: Using replicas, Mezard and Parisi relate the scaling of the PDF for susceptibility with size of a manifold, to its roughness exponent [5]. Exact results on PDFs have been obtained for a directed polymer on a disordered Cayley tree by Derrida and Spohn, using a mapping to a deterministic differential equation [6].

An important example of pinned elastic media is provided by FLs in a superconductor with point impurities. Indeed, our study was motivated by the experiment of Bolle et al. [7] on a 2d FL lattice oriented parallel to a thin micrometer-sized film of $2 \mathrm{H}-\mathrm{NbSe}_{2}$. Magnetic re- sponse measurements show interesting sample-dependent fine structure in $B(H)$; a fingerprint of the underlying pinning landscape. Interestingly, the problem of $2 \mathrm{~d}$ lines with impurities is amenable to an exact solution using replicas, which gives not only the quenched average but also cumulants of the free energy [8]. We shall use this exact solution to motivate an ansatz for the scaling of PDFs in general disorder-dominated thermodynamic systems.

In what follows, we first present a general ansatz for the scaling of the replicated free energy. The key assumption is treating the number of replicas as a scaling field. Consequences of this ansatz for the scaling of cumulants of thermodynamic observables in mesoscopic samples are then enumerated. We then re-examine the example of FLs in a $2 d$ layer in more detail, proposing specific experimental tests of the theoretical picture.

The lack of translational symmetry in the presence of impurities is cured by calculating disorder averaged moments, $\left[Z^{n}\right]$, of the partition function. These moments then provide information about PDFs of the free energy, magnetization, or susceptibility. We thus focus on the scaling of $F_{n}=-T \ln \left[Z^{n}\right]$, the free energy of $n$ replicas of the system, with interactions resulting from the disorder average. In fact $F_{n}$ can be determined exactly for a single elastic line, or a lattice of non-crossing lines, in $2 \mathrm{~d}$ with point impurities [8]. While this is sufficient for the FL experiment [7], we would like to propose a more general scaling ansatz for random systems. Consider $n p$ replicas of a system of size $L_{\|}^{d-1} L_{\perp}$, with $p$ groups of $n$ replicas subject to the same reduced temperature $\tau_{j}$ and scaling field $\psi_{j}$, for $j=1 \ldots p$. The vector of scaling fields $\psi$ can consist of say external magnetic fields, or chemical potentials. Our scaling ansatz for the singular part of the free energy density, $f_{p}(n, \boldsymbol{\tau}, \boldsymbol{\psi})=-T L_{\|}^{-d+1} L_{\perp}^{-1} \ln \left[\prod_{j=1}^{p} Z^{n}\left(\tau_{j}, \psi_{j}\right)\right]$, is

$$
f_{p}^{(\mathrm{s})}(n, \boldsymbol{\tau}, \boldsymbol{\psi})=b^{-d+1-\zeta} f_{p}^{(\mathrm{s})}\left(n b^{\theta_{\|}}, \boldsymbol{\tau} b^{1 / \nu_{\|}}, \boldsymbol{\psi} b^{\delta / \nu_{\|}}\right) .
$$

To include FL systems, we have allowed for anisotropic scaling, with a 'roughness exponent' $\zeta$ relating the $(d-1)$ dimensional longitudinal $\left(l_{\|}\right)$, and the $1 \mathrm{~d}$ transversal $\left(l_{\perp}\right)$ 
scales, by $l_{\perp} \sim l_{\|}^{\zeta}$. (For isotropic systems, we can set $\zeta=1$.) Compared to the standard scaling hypothesis for pure systems, the replica structure introduces $n$ as an additional scaling field with dimension $\theta_{\|}=\zeta \theta_{\perp}$. The exponent $\theta_{\|}$appears in the modified hyperscaling relation $2-\alpha=\nu_{\|}\left(d-1+\zeta-\theta_{\|}\right)$, which follows readily from Eq. (1) by comparing the $\mathcal{O}(n)$ terms. Besides its agreement with available exact results, a mean-field analysis of the $\phi^{4}$-model with Gaussian random fluctuations in $T_{c}$ also supports our ansatz.

Scaling of disorder averaged cumulants of the thermodynamic observables can now be extracted from the above ansatz for $\ln \left[\prod_{j=1}^{p} Z^{n}\left(\tau_{j}, \psi_{j}\right)\right]$. Here we enumerate the main results (details to appear elsewhere):

(1) Free energy cumulants: For $p=1$, we have

$$
\ln \left[Z^{n}(\tau, \psi)\right]=\sum_{j=1}^{\infty} \frac{(-n)^{j}}{j !} \frac{\left[F^{j}(\tau, \psi)\right]_{c}}{T^{j}},
$$

where $[\ldots]_{c}$ denotes the connected or cumulant disorder average. Using a scaling factor proportional to the correlation length, $b \sim \xi_{\|} \sim \tau^{-\nu_{\|}}$, gives $\left[F^{p}(\tau)\right]_{c} \sim$ $\tau^{\nu_{\|}\left(d-1+\zeta-p \theta_{\|}\right)}$. For mesoscopic systems of size $L_{\perp} \sim L_{\|}^{\zeta}$, close to criticality $\left(\xi_{\|}>L_{\|}\right)$we must set $b \sim L_{\|}$, resulting in $\left[F^{p}\right]_{c} \sim L_{\|}^{p \theta_{\|}}$. The exponent $\theta_{\|}$thus characterizes all sample-to-sample fluctuations in the free energy.

(2) Thermal averages, such as the magnetization or the number of 'particles', are first order derivatives of the free energy, which we will denote generally by $\langle X\rangle \equiv$ $-\partial F /\left.\partial \psi\right|_{\psi=0}$. For a specific realization of disorder, the partition function can be expanded as

$$
Z(\psi)=Z(0) \sum_{j=0}^{\infty} \frac{(\psi / T)^{j}}{j !}\left\langle X^{j}\right\rangle,
$$

where $\langle\ldots\rangle$ denotes the thermal average. Using this representation, one can show that the $p^{\text {th }}$ coefficient of the expansion of $\ln \left[Z^{n}(\psi)\right]$ with respect to $n \psi$ is the connected average $\left[\langle X\rangle^{p}\right]_{c} T^{-p} / p$ !. Applying the scaling hypothesis of Eq.(1), we obtain with $\delta=2-\alpha-\beta$ the critical scaling behavior $\left[\langle X\rangle^{p}\right]_{c} \sim \tau^{\beta_{p}}$, with $\beta_{p}=$ $p \beta-\nu_{\|}(d-1+\zeta)(p-1)$, and $\beta$ defined by $[\langle X\rangle] \sim \tau^{\beta}$.

(3) Response functions: Cumulants of the susceptibility, $\chi=\left(\left\langle X^{2}\right\rangle-\langle X\rangle^{2}\right) / T$, can be calculated in a similar way. We now consider $p$ sets of $n$ replicas, each with the same scaling field $\psi_{j}, j=1, \ldots, p$. The coefficient of the term $n^{p}\left(\psi_{1} \cdot \ldots \cdot \psi_{p}\right)^{2}$ of the expansion of the generating function $\ln \left[\prod_{j=1}^{p} Z^{n}\left(\psi_{j}\right)\right]$ is given by the cumulant average $(2 T)^{-p}\left[\chi^{p}\right]_{c}$. This gives a critical scaling of the form $\left[\chi^{p}\right]_{c} \sim \tau^{-\gamma_{p}}$, with $\gamma_{p}=p \gamma+\nu_{\|}(d-1+\zeta)(p-1)$.

(4) Cross correlations, such as $\Gamma_{X Y}=\langle X Y\rangle-\langle X\rangle\langle Y\rangle$, where $Y$ is a derivative of the free energy with respect to another scaling field $\tilde{\psi}$, are also of interest. For example, the cross correlation, $\Gamma_{E M}$, of magnetization $M$ and energy $E$, has been measured in numerical simulations, since it allows for a more accurate estimate of the exponent ratio $\alpha / \nu$ then a direct method [9]. The generator of $\Gamma_{X Y}$ is $\ln \left[\prod_{j=1}^{p} Z^{n}\left(\psi_{j}, \tilde{\psi}_{j}\right)\right]$, with coefficients $T^{2 p}\left[\Gamma_{X Y}^{p}\right]_{c}$ corresponding to the terms $n^{p} \psi_{1} \tilde{\psi}_{1} \ldots \psi_{p} \tilde{\psi}_{p}$. Our scaling ansatz yields $\Gamma_{E M} \sim \tau^{p(\beta-1)-\nu_{\|}(d-1+\zeta)(p-1)}$. There are also correlations between different susceptibilities. Along the lines presented above, it is possible to show that these cross-correlations can be generated from the expansion of $\ln \left[\prod_{j=1}^{p} Z^{n}\left(\psi_{j}\right) Z^{n}\left(\tilde{\psi}_{j}\right)\right]$, and that the cumulant $(2 T)^{-2 p}\left[(\chi \tilde{\chi})^{p}\right]_{c}$ is the coefficient of the term $n^{2 p} \psi_{1}^{2} \tilde{\psi}_{1}^{2} \ldots \psi_{p}^{2} \tilde{\psi}_{p}^{2}$. Choosing for $\chi$ and $\tilde{\chi}$ the magnetic susceptibility and the heat capacity $c$, respectively, we get $\left[(\chi c)^{p}\right]_{c} \sim \tau^{-2 \gamma_{p}-\nu_{\|}(d-1+\zeta)}$.

Finally, we compare with the results of Aharony and Harris [4], on the relative cumulants $R_{p, X}=\left[X^{p}\right]_{c} /[X]^{p}$, in a $\phi^{4}$-model with random $T_{c}$. By a perturbative renormalization group (RG), in which they assume a Gaussian distribution for randomness on all length scales, they obtain $R_{p, \chi}=p ! 2^{p-3} R_{2, \chi}^{p-1}$, for the magnetic susceptibility. Indeed, for all observables (including susceptibilities) we find $R_{p, X} \sim R_{2, X}^{p-1}$, but the exact coefficient cannot be obtained from the scaling ansatz. However, even for an originally Gaussian random $T_{c}$, higher then second cumulants are generated by RG transformations and yield additional (universal) contributions to the above coefficient [4]. Assuming that randomness is relevant at the phase transition, we find for the second relative cumulant of any observable $X$ that $R_{2, X} \sim\left(b / L_{\|}\right)^{d-1}\left(b^{\zeta} / L_{\perp}\right)$. Choosing a scaling factor $b \sim \xi_{\|}$if $\xi_{\|} \ll L_{\|}$, and $b \sim L_{\|}$ in the critical regime $\xi_{\|} \gg L_{\|}$, we reproduce the results of Ref. [4.

We now apply these results to the experimental study by Bolle et al. of a planar, randomly pinned vortex array penetrating a mesoscopic quasi 2-dimensional thin single crystal of $2 \mathrm{H}-\mathrm{NbSe}_{2}$ with weak pinning [7]. The crystal was glued onto a silicon micromachined mechanical resonator. By measuring jumps in the resonant frequency caused by magnetic FLs entering the sample, the number of lines was determined very accurately as a function of the applied magnetic field. Close to the lower critical field $H_{\mathrm{c} 1}$, the jumps were irreversible, indicative of the difficulty of FLs finding optimal pinning configurations. At higher fields, the increased line density should result in a smaller pinning length, enabling the FL lattice to find its optimal pinning state. Indeed reversible behavior is observed experimentally in this case. In both regimes, the response depends on the detailed configuration of pinning sites, the sample geometry, and the vortex interactions. Therefore, the observed discrete jumps in the constitutive relation, $B(H)$, provide a fingerprint of the disorder in a specific sample.

Previous theoretical and experimental work concentrated mainly on the determination of the relation $B(H)$ near $H_{\mathrm{c} 1}$ for different kinds of disorder [10]. Fluctuations 
in $B(H)$ are also of interest, characterizing more clearly the non-trivial effects of randomness in this mesoscopic system. Therefore, we study entire PDFs for observables like $B$, magnetic susceptibility $\chi$, heat capacity $c$, and correlations between them. First consider a single FL to introduce the basic notations. The transversal wandering of such a line is described by a trajectory $x(y)$, which has an energy 11

$$
\mathcal{H}=\int_{0}^{L} d y\left[\frac{g}{2}\left(\frac{d x}{d y}\right)^{2}+V(x(y), y)\right] .
$$

Here, $g=\phi_{0} H_{\mathrm{c} 1} /(4 \pi)$ is the elastic stiffness of the FL carrying a flux quantum $\phi_{0}$. The random potential $V(x, y)$ mimics point like pinning, and is characterized by the disorder average $\left[V(x, y) V\left(x^{\prime}, y^{\prime}\right)\right]=\Delta \delta_{\xi}\left(x-x^{\prime}\right) \delta(y-$ $\left.y^{\prime}\right)$, with strength $\Delta$ and a short range function, $\delta_{\xi}$, of width of the in-plane coherence length $\xi$. The dimensions of the sample are taken to be $L$ along the field direction $\hat{\mathbf{y}}$, and $W$ in the transverse direction $\hat{\mathbf{x}}$.

A single FL can wander freely in the transverse direction to take advantage of the randomly distributed pinning centers. This leads to an anomalous growth of its displacement by $\delta x(L) \sim L^{2 / 3}[12$. In contrast, in a lattice of lines, the non-crossing condition is a strong restriction for the possible configurations of each line. The results of Ref. [8] for such a lattice, only apply to FLs at temperatures $T$ larger than $T^{*}=(g \xi \Delta)^{1 / 3}$, the height of the smallest energy barrier due to the random potential. Recently, Korshunov and Dotsenko [13] generalized the results for a single line to the low temperature limit, $T \ll T^{*}$, by using a replica interaction potential with a small but finite curvature, instead of the rectangular well which works in the high- $T$ limit. The generalization of their solution to an array of lines is straightforward. The non-trivial part of the free energy of $n$ replicas of the system, with a fixed number of $N=W / a_{0}$ of lines at mean separation $a_{0}$, can be summarized in both limits as [8]

$$
\frac{F_{n}(N)}{T}=-\ln \left[Z^{n}\right]=n k N^{2} \frac{L}{W} \frac{\Delta}{T^{2}} \mathcal{G}\left(n \sqrt{\frac{k g \Delta W}{N T^{3}}}\right),
$$

with the parameter $k=1$ for $T \gg T^{*}$, and $k \approx T / T^{*}$ for $T \ll T^{*}$. Here we have neglected the trivial contribution to the free energy which is linear in $N$, and $\mathcal{G}$ is an analytic function 14 .

To study the fluctuations in the number of FLs near $H_{\mathrm{c} 1}$, we use a grand canonical description with a chemical potential $\mu=g h L$, where $h=\left(H-H_{\mathrm{c} 1}\right) / H_{\mathrm{c} 1}$ is the reduced magnetic field. The PDF of $N$ is characterized by the disorder averaged cumulants $\left[N^{p}\right]_{c}$ in the number of FLs, which are given by $T^{p}$ times the coefficients of the terms $(n \mu)^{p}$ in the expansion of $\ln \left[Z^{n}(\mu)\right]$, leading to

$$
\left[N^{p}\right]_{c} \sim W L^{1-p}\left(\frac{g T}{k \Delta}\right)^{2-p} h^{(5-3 p) / 2} .
$$

The moments of the magnetic flux density follow from $B=\phi_{0} N /(d W)$, where $d$ is the thickness of the sample. It is interesting to note that the second cumulant is universal, $\left[N^{2}\right]_{c} \sim(W / L) h^{-1 / 2}$, independent of temperature and disorder strength, in both the high and low temperature regimes. For $T \ll T^{*}$, all cumulants are independent of $T$, but do depend on the disorder strength. In the thermodynamic limit $W L \rightarrow \infty$, all cumulants $\left[B^{p}\right]_{c}$ approach zero for $p>1$. On the other hand, for mesoscopic systems, the divergence of $\left[B^{p}\right]_{c}$ for $h \rightarrow 0$ is stopped if the average distance $a_{0}$ between the FLs approaches the system size $W$. Deep in the glassy low density phase near $H_{\mathrm{c} 1}$, we obtain from Eq. (5) for the free energy cumulants

$$
\left[F^{p}\right]_{c} \sim W L g^{2}\left(\frac{T}{k \Delta}\right)^{2-p} h^{(5-p) / 2} .
$$

This agrees with our scaling ansatz with exponents $\zeta=$ $2 / 3, \theta_{\|}=1 / 3, \nu_{\|}=3 / 2$ and $\delta=1$, and gives a universal value for $p=2$, which still depends on the stiffness $g$.

Now consider the response of the FL lattice to changes in the external magnetic field, measured by the susceptibility $\chi$. This is related to thermal fluctuations in the number of FLs by $\chi=(L / W)\left(\left\langle N^{2}\right\rangle-\langle N\rangle^{2}\right) / T$, for a fixed realization of disorder. The disorder averaged $p^{\text {th }}$ cumulant of the number fluctuations can be obtained from the generating function $\ln \left[\prod_{j=1}^{p} Z^{n}\left(\mu_{j}\right)\right]$ for $p$ different chemical potentials $\mu_{j}$, giving the susceptibility cumulants

$$
\left[\chi^{p}\right]_{c} \sim(W L)^{1-p} g^{2-2 p}\left(\frac{T}{k \Delta}\right)^{2-p} h^{-5(p-1) / 2} .
$$

This result deserves a few comments: First, the disorder averaged susceptibility $[\chi]$ is non-singular at the transition $h \rightarrow 0$, since the exponent vanishes for $p=1$. In fact, due to a statistical tilt symmetry [15] the susceptibility is simply related to the compression and tilt elastic moduli $\left(c_{11}, c_{44}\right)$ of the FL lattice, by $[\chi]=\left(2 \pi / a_{0}\right)^{2}\left(c_{11} c_{44}\right)^{-1 / 2}$. This is in agreement with our result $[\chi] \sim T /(k \Delta)$, as can be seen by using $c_{44}=g / a_{0}$, rewriting $c_{11}$ in terms of the steric repulsion between the lines, and estimating $a_{0}=W / N$ from Eq. (6) with $p=1$, and a value for $h$ of order one. Again, the variance is the only moment of the susceptibility, which shows universality, $\left[\chi^{2}\right]_{c} \sim\left(W L g^{2}\right)^{-1} h^{-5 / 2}[16]$.

Next, consider the response of a fixed number of FLs to changes in temperature. Keeping the number $N$ of FLs constant by adjusting the magnetic field, but changing the temperature, allows us to study thermal fluctuations around the global ground-state. Physically, the response of the FL lattice to changes in magnetic field $H$, or temperature $T$, are quite different: A change in $H$ (or $N$ ) usually leads to a complete rearrangement of the whole ensemble of lines. Increasing $T$, however, causes stronger 
entropic repulsions between the $N$ lines, which now fluctuate around their state of optimal pinning. The response to a change in $T$ also depends on the detailed pinning landscape, producing a heat capacity $c$ which is sample specific. Therefore, the statistics of $c$ are also of interest; its cumulants can be obtained from the generating function $\ln \left[\prod_{j=1}^{p} Z^{n}\left(\beta_{j}\right)\right]$ with $p$ different temperatures $T_{j}=1 / \beta_{j}$, as (for fixed $\left.N\right)$

$$
\left[c^{p}\right]_{c} \sim L W^{\frac{p-3}{2}}(k \Delta)^{\frac{p+1}{2}} g^{\frac{p-1}{2}} N^{\frac{5-p}{2}} T^{-\frac{3 p+1}{2}} .
$$

In the high temperature limit $\left(T \gg T^{*}\right)$, the moments $\left[c^{p}\right]_{c} \sim T^{-(3 p+1) / 2}$ decay faster with temperature than in the low temperature limit $\left(T \ll T^{*}\right)$, where $\left[c^{p}\right]_{c} \sim T^{-p}$.

We may also examine cross-correlations between the different susceptibilities such as the heat capacity $c$, and the magnetic susceptibility $\chi$. In the thermodynamic limit, i.e. beyond a characteristic system size, different susceptibilities are expected to be statistically independent. For example, this has been demonstrated for the magnetic susceptibilities of two noninteracting FL lattices with different random potentials 15. The correlations between $c$ and $\chi$ can be obtained from our scaling theory by extracting the coefficients of $n^{2 p} \mu_{1}^{2} \beta_{1}^{2} \ldots \mu_{p}^{2} \beta_{p}^{2}$ of $\ln \left[\prod_{j=1}^{p} Z^{n}\left(\mu_{j}\right) \prod_{j=1}^{p} Z^{n}\left(\beta_{j}\right)\right]$. Using the results of Eqs. (8) and (9), we get

$$
\frac{\left[(\chi c)^{p}\right]_{c}}{\left[\chi^{p}\right]_{c}\left[c^{p}\right]_{c}} \sim(W L)^{-1}\left(\frac{k \Delta}{g T}\right)^{2} h^{-5 / 2} .
$$

For finite systems, the divergence as $h \rightarrow 0$ is cut off at $h \sim k \Delta /(g T W)$. Then the result can be rewritten as $\left[(\chi c)^{p}\right]_{c} /\left(\left[\chi^{p}\right]_{c}\left[c^{p}\right]_{c}\right) \sim L_{c} / L$, with a characteristic length scale $L_{c}=(g T / k \Delta)^{1 / 2} W^{3 / 2}$ for the decay of correlations. This length-scale has a simple physical interpretation: $L_{c}$ is the length of a single FL whose transverse wanderings can explore the whole sample width $W$. Since the transversal fluctuations of a line of length $L$ is grow as $\delta x(L) \approx(k \Delta / g T)^{1 / 3} L^{2 / 3}[13$, we get the above result for $L_{c}$ from the condition $\delta x\left(L_{c}\right) \approx W$.

The full shape of the PDFs is determined by the relative cumulants $R_{p, X}=\left[X^{p}\right]_{c} /[X]^{p}$. For $X=N, \chi, c$ or $\chi c$, our results yield $R_{p, X} \sim R_{2, X}^{p-1}$ up to a numerical coefficient. Therefore, the system parameters enter the PDF shapes only through $R_{2, X}$, which is interestingly independent of the observable $X$, and given by

$$
R_{2, X} \sim(W L)^{-1}\left(\frac{k \Delta}{g T}\right)^{2} h^{-5 / 2}=\frac{\xi_{\perp} \xi_{\|}}{W L} .
$$

The length scales in the final expression are $\xi_{\perp}=a_{0}$, the separation of FLs, and $\xi_{\|}=(g T / k \Delta)^{1 / 2} a_{0}^{3 / 2}$, the mean longitudinal distance between collisions of FLs. To obtain a size independent PDF, anisotropy requires looking at finite-size samples of width $W \sim L^{\zeta}$, with roughness exponent $\zeta=2 / 3$. The resulting $\mathrm{PDF}$ is however, still non-universal, depending on $R_{2, X} \sim(g T / k \Delta)^{1 / 2}$.
The above predictions for the scaling of PDFs, or cumulants, can be tested experimentally by measurements on different realizations of randomness, drawn from the same distribution of impurities. Generating many such different realizations could in fact be quite easy, depending on the system under study. For example, in the case of the FL lattice of Ref. [7] experiments can be performed on the same sample, with different realizations of randomness generated by simply rotating the sample with respect to the external magnetic field. Each (finite-size) realization of randomness yields a characteristic value for thermodynamic observables, providing a reproducible 'fingerprint' of the configuration. The statistics of these measurements is then described by the cumulants calculated here.

We thank D.R. Nelson for bringing the experimental system of Ref. [7] to our attention. This work was supported by the Deutsche Forschungsgemeinschaft under grant EM70/1-1 (TE), and the National Science Foundation grant No. DMR-98-05833 (MK).

[1] Mesoscopic Quantum Physics edited by E. Akkermans et al. (North-Holland, Amsterdam, 1994).

[2] T. Davis and J. Cardy, preprint cond-mat/9911083.

[3] T. Nattermann, Int. J. Mod. Phys. B 3, 1597 (1989).

[4] A. Aharony, A.B. Harris, Phys. Rev. Lett. 77, 3700 (1996).

[5] M. Mezard and G. Parisi, J. Phys. I (Paris) 1, 809 (1991).

[6] B. Derrida and H. Spohn, J. Stat. Phys. 51, 817 (1988).

[7] C.A. Bolle et al., Nature 399, 43 (1999).

[8] M. Kardar, Nucl. Phys. B 290, 582 (1987).

[9] H.O. Heuer, Phys. Rev. B 42, 6476 (1990).

[10] For a recent detailed summary of the theoretical results see: R.A. Lehrer and D.R. Nelson, preprint condmat/9908117.

[11] D.R. Nelson, Phys. Rev. Lett. 60, 1973 (1988); T. Nattermann and R. Lipowsky, Phys. Rev. Lett. 61, 2508 (1988).

[12] D.A. Huse, C.L. Henley, and D.S. Fisher, Phys. Rev. Lett. 55, 2924 (1985).

[13] S.E. Korshunov, V.S. Dotsenko, J. of Phys. A 312591 (1998).

[14] The result of Eq. (5) is valid for systems of size $W \gg$ $T^{3} /\left(k^{3} g \Delta\right)$. For $2 \mathrm{H}-\mathrm{NbSe}_{2}$, in the relevant regime of $T \ll T^{*}$, this condition becomes $W \gg \xi$, which is fulfilled even for mesoscopic samples.

[15] T. Hwa, D.S. Fisher, Phys. Rev. Lett. 72, 2466 (1994).

[16] Universal sample-to-sample fluctuations of the magnetic susceptibility have been predicted in this $2 \mathrm{~d}$ FL system at the so-called glass transition in Ref. [15], and were confirmed by numerical simulations in C. Zeng, P.L. Leath, and T. Hwa, Phys. Rev. Lett. 83, 4860 (1999). 